\title{
What is hypertension?
}

\section{Genevieve Gabb}

Senior staff specialist, Acute and Urgent Care, Royal

Adelaide Hospital

Consultant, Cardiology,

Division of Medicine,

Cardiac and Critical Care,

Flinders Medical Centre,

Bedford Park, SA

Clinical senior lecturer,

Discipline of Medicine

School of Medicine,

Faculty of Health Science,

University of Adelaide

Senior lecturer, College of

Medicine and Public Health,

Flinders University, Bedford

Park, SA

\section{Keywords}

antihypertensive drugs,

blood pressure,

cardiovascular risk

hypertension

Aust Prescr 2020;43:108-9

https://doi.org/10.18773/ austprescr.2020.025
Hypertension is both a disease and a major risk factor for other diseases. Population studies show an increasing rate of cardiovascular events such as stroke, myocardial infarction, heart failure, atrial fibrillation and premature mortality, with increasing blood pressure (from systolic blood pressures $\geq 115 \mathrm{mmHg}$ ). This relationship is exponential, and stronger for systolic pressure than for diastolic pressure.

Untreated very high ( $>180 / 110 \mathrm{mmHg}$ ) or rapidly rising blood pressure (such as in eclampsia) can overcome normal microvascular autoregulation. This leads to acute damage in the microcirculation and results in a multisystem clinical syndrome of accelerated or malignant hypertension, or cerebral haemorrhage, which are immediate threats to life. ${ }^{1}$ Accelerated or malignant hypertension is now fortunately uncommon. The main consideration in the majority of individuals is the relationship between their blood pressure and subsequent risk of cardiovascular disease. Given the continuous relationship of blood pressure to risk, any level of blood pressure used to define 'hypertension' will always be arbitrary. The critical issue is, how do we define hypertension, and does it matter?

International guidelines for the management of hypertension have been published for more than 40 years. The most recent updates are from Australia (2016), ${ }^{2}$ the USA (2017), ${ }^{3}$ Canada (2018), ${ }^{4}$ Europe $(2018)^{5}$ and the UK (2019). ${ }^{6}$ In defining hypertension, these guidelines have taken two approaches, either basing their definition on a threshold for treatment, or alternatively on the blood pressure above which the risk of events is increased.

The Australian, Canadian, European and UK guidelines have chosen a cut-off level of blood pressure above which the benefits of treatment, demonstrated by interventional clinical trials of blood pressure lowering therapy, are considered to outweigh the harms of treatment. Using this approach, the cut-off point which defines hypertension is $140 / 90 \mathrm{mmHg}$ using standard clinic methods of measurement. These guidelines also emphasise the range of severity of hypertension by stratifying blood pressure above the cut-off point. Hypertension, using this grading scheme, is defined as:

- grade 1-140-159 mmHg systolic or 90-99 $\mathrm{mmHg}$ diastolic

- $\quad$ rade 2 - 160-179 mmHg systolic or 100-109 mmHg diastolic

- $\quad$ grade 3-180 mmHg systolic or $110 \mathrm{mmHg}$ diastolic and above. ${ }^{2}$
The benefit of lowering blood pressure to prevent hypertension-related disease and reduce cardiovascular events and mortality is unequivocal in patients with very substantial elevations in blood pressure. This was reported in 1967 with publication of the first randomised controlled interventional study of antihypertensive therapy in patients with diastolic blood pressures averaging 115-129 mmHg. ${ }^{7}$ Subsequent studies, which have mostly used measures of clinic blood pressure, have shown the benefit of blood pressure lowering when the systolic pressure is above $140 \mathrm{mmHg}$ in all patients up to age 80 years ${ }^{8,9}$ and above $160 \mathrm{mmHg}$ in those over 80 years. $^{10}$ The relative risk reduction is similar across the range of baseline cardiovascular risk. Patients with the highest baseline risk have the greatest absolute benefit (lowest numbers needed to treat to prevent an event)."

In contrast, the US guideline has redefined hypertension, for both clinical and public health decision making, on the degree of blood pressure elevation associated with increased cardiovascular risk (hazard ratio 1.5-2.0). The US categories for stage 1 hypertension are systolic blood pressures of 130-139 mmHg or diastolic blood pressures of $80-89 \mathrm{mmHg}$ and stage 2 is systolic blood pressures of $140 \mathrm{mmHg}$ and above. These categories are substantially lower than in the threshold-based guidelines, and do not distinguish risks at levels above $140 \mathrm{mmHg}$, despite the known exponential increased risk with increasing blood pressure.

A consideration with the US definition is that current evidence from interventional studies does not show a benefit from starting blood pressure lowering therapy if the untreated systolic blood pressure is below $140 \mathrm{mmHg}$ in individuals without cardiovascular disease. ${ }^{5}$ There may possibly be benefit from blood pressure lowering at a lower baseline blood pressure only in those with the highest cardiovascular risk and established cardiovascular disease. ${ }^{5}$ The majority view at present is therefore that the definition of hypertension is best based operationally on an evidence-based treatment threshold. 2,4,5,6

Blood pressure is highly variable within an individual, and is not well characterised from a single or very few measurements. Historically, a diagnosis of hypertension in the majority of interventional trials has been based on repeated clinic measures taken on multiple occasions. There are now additional approaches for measuring the blood pressure 
profile over 24 hours (non-invasive ambulatory blood pressure) or over a longer time (home blood pressure monitoring) that are more closely linked to cardiovascular outcomes. ${ }^{12}$ These methods result in readings that are lower than clinic measurements which must be considered when making a diagnosis of hypertension. A daytime ambulatory or home blood pressure of $135 / 85 \mathrm{mmHg}$ is approximately equivalent to a clinic blood pressure of $140 / 90 \mathrm{mmHg} .^{12} \mathrm{An}$ alternative approach initially promoted in Canada is that of using automated measurements of blood pressure in the clinic. This approach results in readings that are lower than usual clinic blood pressures, but very similar to the average daytime reading from a 24-hour ambulatory monitor. However, this approach has not yet been widely adopted internationally and importantly is not the method of blood pressure measurement recommended for use in current cardiovascular risk calculators. ${ }^{2}$

Why bother with a definition of hypertension, given the continuous nature of the relationship between blood pressure and risk, and the difficulties with measurement? Arbitrarily defining hypertension as being an average sustained clinic blood pressure of $140 / 90 \mathrm{mmHg}$ or above is clinically useful as it clearly identifies a level of blood pressure where individuals, if untreated and without established vascular disease, could benefit from blood pressure lowering therapy and should be offered it. Clinicians can be confident this definition is supported with clinical trial evidence. Grade 2 hypertension above this clearly identifies increasing risk with increasing blood pressure, reflecting the known exponential relationship between blood pressure and vascular outcomes and an even stronger imperative for treatment. Individual treatment decisions are, however, more complex than a definition. Fortunately, there is a range of excellent guidelines on hypertension to support these. ${ }^{2-6}<$

Genevieve Gabb is a member of the Executive Committee, High Blood Pressure Research Council of Australia, and a member of the Clinical Committee, Heart Foundation of Australia.

\section{REFERENCES}

1. Kincaid-Smith P, McMichael J, Murphy EA. The clinical course and pathology of hypertension with papilloedema (malignant hypertension). Q J Med 1958;27:117-54. https://doi.org/10.1093/oxfordjournals.qjmed.a066802

2. Gabb GM, Mangoni AA, Anderson CS, Cowley D, Dowden JS, Golledge J, et al. Guideline for the diagnosis and management of hypertension in adults - 2016. Med J Aust 2016;205:85-9. https://doi.org/10.5694/mja16.00526

3. Whelton PK, Carey RM, Aronow WS, Casey DE Jr, Collins KJ, Dennison Himmelfarb C, et al. 2017 ACC/AHA/AAPA/ABC/ ACPM/AGS/APhA/ASH/ASPC/NMA/PCNA Guideline for the prevention, detection, evaluation, and management of high blood pressure in adults: a report of the American College of Cardiology/American Heart Association Task Force on Clinical Practice Guidelines. J Am Coll Cardiol 2017;71:e127-248. https://doi.org/10.1016/j.jacc.2017.11.006

4. Hypertension Canada. Hypertension Canada Guidelines [Internet]. https://guidelines.hypertension.ca [cited 2020 Aug 1]

5. Williams B, Mancia G, Spiering W, Agabiti Rosei E, Azizi M, Burnier M, et al. 2018 ESC/ESH Guidelines for the management of arterial hypertension. Eur Heart J 2018;39:3021-104. https://doi.org/10.1093/eurheartj/ehy339

6. National Institute for Health and Care Excellence. Hypertension in adults: diagnosis and management. NICE guideline [NG136]. London: NICE; 2019. https://www.nice.org.uk/guidance/ng136 [cited 2020 Aug 1]

7. Veterans Administration Cooperative Study Group on antihypertensive agents. Effects of treatment on morbidity in hypertension. Results in patients with diastolic blood pressures averaging 115 through 129 mm Hg. JAMA 1967;202:1028-34. https://doi.org/10.1001/jama.1967.03130240070013
8. Sundström J, Arima H, Jackson R, Turnbull F, Rahimi K, Chalmers J, et al. Effects of blood pressure reduction in mild hypertension: a systematic review and meta-analysis. Ann Intern Med 2015;162:184-91. https://doi.org/10.7326/ M14-0773

9. Lonn EM, Bosch J, López-Jaramillo P, Zhu J, Liu L, Pais P, et al. Blood-pressure lowering in intermediate-risk persons without cardiovascular disease. N Engl J Med 2016;374:2009-20. https://doi.org/10.1056/NEJMoa1600175

10. Becket NS, Peters R, Fletcher AE, Staessen JA, Liu L, Dumitrascu D, et al. Treatment of hypertension in patients 80 years of age or older. N Engl J Med 2008;358:1887-98. https://doi.org/10.1056/NEJMoa0801369

11. Blood Pressure Lowering Treatment Trialists' Collaboration. Blood pressure-lowering treatment based on cardiovascular risk: a meta-analysis of individual patient data. Lancet 2014;384:591-8. https://doi.org/10.1016/S0140-6736(14)61212-5

12. Sega R, Facchetti R, Bombelli M, Cesana G, Corrao G, Grassi G, et al. Prognostic value of ambulatory and home blood pressures compared with office blood pressure in the general population: follow-up results from the Pressioni Arteriose Monitorate e Loro Associazioni (PAMELA) study. Circulation 2005;111:1777-83. https://doi.org/10.1161/ 01.CIR.0000160923.04524.5B 